\title{
Visualizing Virus Assembly Intermediates Inside Marine Cyanobacteria by Zernike Phase Contrast Electron Cryo-Tomography
}

Wah Chiu ${ }^{1,2, *}$, Wei Dai ${ }^{1}$, Caroline $\mathrm{Fu}^{1}$, Desislava Raytcheva ${ }^{3,4}$, John Flanagan $^{1, \dagger}$, Htet A. Khant ${ }^{1}$, Xiangan Liu ${ }^{1}$, Ryan H. Rochat ${ }^{1,2}$, Cameron Haase-Pettingell ${ }^{3}$, Jacqueline Piret ${ }^{4}$, Steve J. Ludtke ${ }^{1,2}$, Kuniaki Nagayama ${ }^{5}$, Michael F. Schmid ${ }^{1,2}$, and Jonathan A. King ${ }^{3}$

1. National Center for Macromolecular Imaging, Verna and Marrs Mclean Department of Biochemistry and Molecular Biology, Baylor College of Medicine, Houston, TX, USA.

2. Program in Structural and Computational Biology and Molecular Biophysics, Baylor College of Medicine, Houston, TX, USA.

3. Department of Biology, Massachusetts Institute of Technology, Cambridge, MA, USA.

4. Department of Biology, Northeastern University, Boston, MA, USA.

5. National Institute for Physiological Sciences, National Institutes of Natural Sciences,

5-1 Higashiyama, Myodaiji, Okazaki, Japan

${ }^{\dagger}$ Present address: FEI, 5350 Dawson Creek Drive, Hillsboro, OR, USA

Cyanobacteria are photosynthetic organisms responsible for $\sim 25 \%$ of organic carbon fixation on the Earth. These bacteria began to convert solar energy and carbon dioxide into bioenergy and oxygen more than two billion years ago. Cyanophages that infect these bacteria have an important role in regulating the marine ecosystem by controlling cyanobacteria community organization and mediating lateral gene transfer among hosts. A key question in phage pathogenesis is the coordination between protein shell assembly and genome packaging. Here, we used Zernike phase contrast (ZPC) electron cryo-tomography (CryoET) to follow the maturation process of wild-type cyanophage Syn5 as it occurs inside its host, Synechococcus sp. WH8109. Zernike phase plates used in our study shift the phase of the scattered electrons by $\pi / 2$, and significantly enhance the low-frequency information, allowing for in-focus, high contrast imaging [1-4]. Consequently, low-contrast features difficult to detect in conventional cryoET images can be more readily identified, and thus facilitates the direct identification of subcellular components, including thylakoid membranes, carboxysomes and polyribosomes, as well as phages, inside the congested cytosol of the infected cell (Figure 1).

Cyanophage Syn5 that infects WH8109 cells is a short-tailed icosahedral phage with a unique horn appendage at the vertex opposite to the tail $[5,6]$. By correlating the structural features and relative abundance of viral progeny within cells at different stages of infection, we identified five distinct Syn5 assembly intermediates: procapsids with scaffolding protein inside the particle, expanded phages with terminase at one of the 12 vertices, DNA-containing phages, phages with fully packed DNA and a tail at one vertex, and mature phages with a tail at one vertex and a horn at the opposite vertex. Our results indicate that the procapsid releases scaffolding proteins and expands its volume at an early stage of genome packaging. Later in the assembly process, the detection of full particles with a tail either with or without an additional horn suggests that the horn is added to the capsid as the final step of assembly. The morphogenetic pathway we describe here is highly conserved and was probably established long before that of double-stranded DNA viruses infecting more complex organisms.

The value of our imaging approach lies in its potential to study the transient biological processes in their natural intracellular environment at nanometer resolution. ZPC cryoET enables direct capturing of structural snapshots of phage assembly intermediates during maturation process. Such analysis can be 
generalizable not only to human viruses in human cells but also various molecular machines undergoing biological processes in the cellular context. Combined with post-tomographic analyses [7], this approach allowed us to mine the rich trove of spatial and temporal information conveyed by the complex biological process of phage infection and maturation in situ.

\section{References:}

[1] R Danev and K Nagayama, Methods Enzymol. 481 (2010), p. 343.

[2] K Murata et al., Structure 18 (2010), p. 903.

[3] R Danev, RM Glaeser and K Nagayama, Ultramicroscopy 109 (2009), p. 312.

[4] M Marko et al, J Struct Biol 174 (2011), p. 400.

[5] WH Pope et al, J. Mol. Biol. 368 (2007), p. 966.

[6] DA Raytcheva et al, J. Virol. 85 (2011), p. 2406.

[7] MF Schmid and CR Booth, J. Struct. Biol. 161 (2008), p. 243.

[8] This research was supported by grants from Robert Welch Foundation (Q1242) and National Institutes of Health (P41GM123832 to W.C.; AI0175208 and PN2EY016525 to W.C. and J.A.K.; GM080139 to S.J.L.; T15LM007093 through the Gulf Coast Consortia to W.D. and R.H.R.; T32GM007330 through the MSTP to R.H.R.).

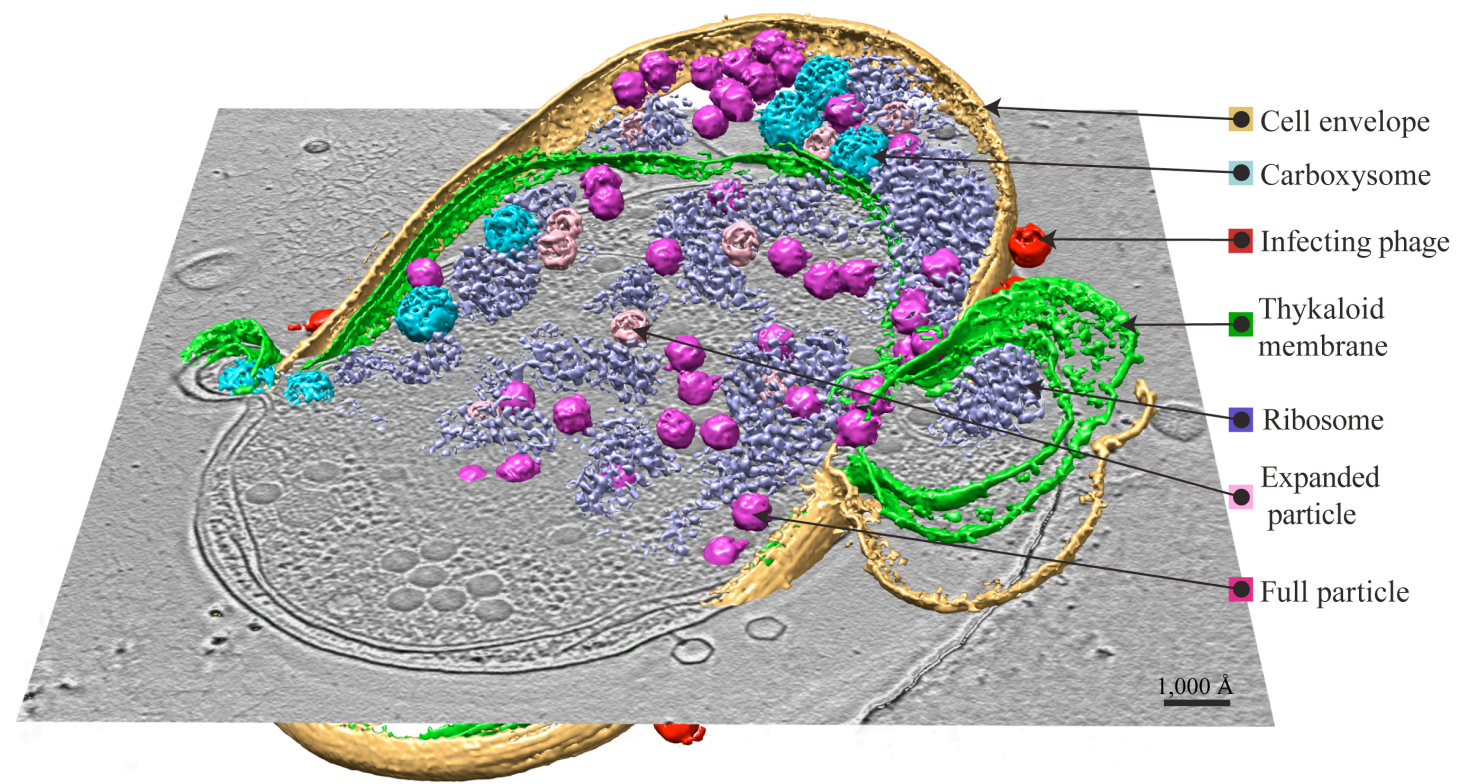

Figure 1. 3-D image shows the structure of a cyanobacteria infected with phages, along with a slice through the center of the structure. After infecting the cell, the phage uses the bacteria's own processes to produce new phages (magenta and peach), which in turn infect additional cells. Zernike phase plates allow us to visualize this process in detail for the first time. 